Herrn

Dr. Gustáv Husák

Präsident der Tschechoslowakischen

sozialistischen Republik

PRAHA - HRAD

Prag - Brünn, den r. März 1976

Betr.: Ersuchen um Freilassung tschechischer politischer Gefangener

Beschwerde über ihre unmenschlichen, gesetzwidrigen Lebensbedingungen.

Herr Präsident,

im Einklang mit der Verfassung der CSSR wenden wir uns an Sie mit dem Ersuchen, sich aufgrund Ihrer Vollmachten für die Freilassung unserer Söhne, Gatten und Väter einzusetzen, die wegen ihrer politischen Ansichten und Haltungen, die ihre sozialistische Uberzeugung ausdrücken, eingekerkert sind.

Gleichzeitig legen wir Ihnen die Beschwerde über ihre Lebensbedingungen vor, die im Widerspruch mit dem Internationalen Pakt über bürgerliche und politische Rechte, mit der Verfassung der CSSR und mit dem Gesetz über den Strafvollzug bei Freiheitsstrafen stehen.

Die Lebensbedingungen tschechischer politischer Häftlinge zielen nämlich auf ibre psychische Brechung und ibre physische Vernichtung!

Die Unterbringung

Obwohl unsere Verwandten in die mildeste erste Gruppe verurteilt wurden, sind sie in Wirklichkeit unter den schlimmsten Haftbedingungen untergebracht. Bereits seit langen Jahren (während der Untersuchungshaft sowie bei dem Strafvollzug) werden sie gezwungen, in kleinen, ständig verschlossenen Zellen von einem Ausmaß von ungefähr $2 \times_{4} \mathrm{~m}$ zu leben, in dauerhafter Isolierung gemeinsam mit einem Mithäftling, der in der Regel ein »Nichtpolitischer « ist. Das Zusammenleben mit einem einzigen Menschen gestaltet sich bei einem tagtäglichen vierundzwanzigstündlichen zwangsweisen Beisammensein zu einer Belastung, die alle Kräfte in Anspruch nimmt. Die Kommunikation mit dem Mithäftling wird darüber hinaus durch die Rücksicht auf die Abhörapparaturen beeinflußt, die von der Gefängniszelle in den Raum des Gefängniskommandanten führen.

Unsere Verwandten sind einer langfristigen und grausamen Verarmung sinnlicher Eindrücke unterworfen: ihre Welt entbehrt der Natürlichkeit der Farben, der Töne, der übrigen Eindrücke. Die Armseligkeit, die beinahe völlige Absenz neuer Anregungen der Sinne, die langfristige Isolierung und die zwangsweise Gesellschaft eines Mitgefangenen - dies alles ist der erste der Umstände, die auf die Brechung des Menschen zielen.

Die Arbeit

Die Gefangenen arbeiten in den Zellen: sie nähen Knöpfe auf Kartons an, sie sortieren und fädeln Sicherheitsnadeln ein, oder Haarnadeln, Patentknöpfe, sie

\footnotetext{
* Es geht um die politischen Gefangenen Jiri Müller - I968 Studentensprecher, Dr. Jan Tesar - Historiker, Dr. Milan Hübl - Historiker, 1968 Mitglied des ZK der KPC und Rektor der Parteihochschule, Unıy. Prof. Dr. Jaroslav Sabata - Politologe, I968 Mitglied des ZK der KPC, Ing. Antonın Rusek - Okonom, 1968 Abgeordneter des T'schechischen Natıonalrates. Eine ausführliche, die in dem Brief enthaitenen Vorwürfe belegende Dokumentation ist enthalten in: Listy-Blätter, Junı 1976, S. 5-12; zu beziehen über: Listy-Blätter, 6 Ffm I Postfach 3602.
} 
sortieren Glasperlen, erzeugen Kunstblumen, flechten Stühle und ähnliches. Das gemeinsame Merkmal aller dieser Arbeiten ist die Monotonie und das hohe Arbeitstempo, das es manchmal erfordert, auch 10-1 2 Stunden täglich zu arbeiten. Die Nichterfüllung der Norm wird bestraft - beispielsweise durch die Beschränkung der Kostzuteilung. Man nimmt keine Rücksicht auf Hindernisse, die durch die Gefangenen selbst nicht verursacht wurden.

Das Gesetz verankert die Arbeitspflicht mit Rücksichtnahme auf den Gesundheitszustand und die Arbeitsfähigkeiten des Gefangenen und fordert dazu auf, Bedingungen zur Erhöhung seiner Qualifikation zu bilden. Die tschechischen politischen Gefangenen haben Berufe meist mit Hochschulbildung (Geschichte, Okonomie, Psychologie, Soziologie, Maschinenbau, Medizin...), die man im Gefängnis schwer zur Geltung bringen kann, die Gefängnisse respektieren jedoch den Geist des Gesetzes nicht einmal durch ein Anzeichen einer Bestrebung, den Gefangenen wenigstens ihre Gesundheit und ihre berufliche Qualifikation aufrecht zuerhalten. Die Arbeitsbedingungen werden durch die fahrlässige Arbeitshygiene, die bis zu einer grausamen Absichtlichkeit reicht, noch verschärft.

Der Lohn politischer Gefangener beträgt nicht einmal die Hälfte des Verdienstes der übrigen (nichtpolitischen, das heißt kriminellen) Gefangenen und etwa ein Fünftel des Durchschnittlohnes in der CSSR. Dieser Lohn erreicht das tschechoslowakische Lebensminimum nicht, nichtsdestotrotz zieht das Gefängnis für eigene Zwecke mehr als $80 \%$ des eigenen Lohnes ab. Der Gefangene erhält von seinem Lohn ungefähr $20-40 \mathrm{Kcs}$ monatlich, und das ist das einzige Geld, für welches er seine Speisekarte ergänzen darf (denn von Zuhause darf er kein Geld erhalten).

Es handelt sich also um eine mehrfache Ausbeutung: bei der Festsetzung der Arbeitsnorm, bei der Bemessung des Erfüllens der Arbeitsnorm und bei den Lohnabzügen. Solche Arbeitsbedingungen mit Leibeigenschaft sind mit unserer Gesellschaftsordnung unvereinbar. Damit wird die Verfassung der CSSR verletzt, die jedwede Ausbeutung ausschließt.

Die Gesundheitspllege

Diejenigen, die bereits aus dem Gefängnis zurückkehrten, kamen mit einer zerrütteten Gesundheit heraus und waren gezwungen, eine gewisse Zeit in der Rekonvaleszens zu verbringen. Vielen wurde im Gefängnis das Augenlicht unwiederherstellbar verdorben. Von denjenigen, die noch gefangengehalten werden, ist keiner mehr so gesund, wie er bei der Einlieferung ins Gefängnis war. Manche sind schwer krank, andere leiden an chronischen Beschwerden.

Die Gesundheit der Gefangenen wird am meisten durch den totalen Mangel an frischer Luft, an Licht, an Bewegung und durch die schlechten hygienischen Möglichkeiten allgemein, durch die schlechte Arbeitshygiene, weiter durch die proteinarme Kost, in der Mineralstoffe und Vitamine fehlen, vernichtet. Die Beköstigung im Gefängnis läßt die Grundsätze der richtigen Ernährung völlig außer Acht. Das Päckchen von Zuhause, bewilligt einmal in drei Monaten im Gewicht bis zu $3 \mathrm{~kg}$, ist außerstande, alles das zu ersetzen, was bei der Beköstigung fehlt. Wenn das Päckchen dem Gefangenen strafweise verboten wird, dann leidet der Gefangene an einer groben Unterernährung - er leidet durch Proteinhunger. Die Beschaffung der Einkäufe in der Kantine wird den politischen Häftlingen erschwert.

Die ärztliche Betreuung gewährt das Gefängnis oft widerstrebend und die vom Arzt erteilten Ratschläge werden meist nicht respektiert. Die Anzeichen der Krankheiten werden mißachtet, so daß die kleineren Beschwerden leicht in größere überwachsen, chronisch werden oder eine gewichtigere Gesundheitsstörung entstehen lassen. Die Heilung der Krankheiten wird auch durch die private Initiative einzelner Wachleute erschwert. 
Die ärztliche Behandlung politischer Gefangener heilt im besten Falle die Beschwerden selbst, es fehlt jedoch völlig die Prävention. Sie fehlt aber nicht nur, die gesundheitlichen Schädigungen werden sogar durch das gesamte System des Strafvollzuges künstlich hervorgerufen. Dadurch verstößt man gegen die Verfassung der CSSR.

Die soziale Situation des Gefangenen und serner Familie

Obwohl das Gerichtsurteil nur den Gefangenen betraf, wird gemeinsam mit ihm auch seine gesamte Familie schwerwiegend geschädigt. Die Kinder können ihren Vater insgesamt nur vier Stunden in einem Jahr sehen und hören - meist dürfen sie ihn nicht einmal berühren. (Der Besuch wird einmal vierteljährlich für eine Stunde für zwei Erwachsene aus den Reihen der engsten Verwandten und für die eigenen Kinder bewilligt. Die Kerkermeister bemühen sich, die Besuche in größtmöglichem Ausmaß zu erschweren und unangenehm zu gestalten. Deshalb kommen vor allem bei älteren Personen in der Zeit vor dem Besuch rätselhafte Verschlechterungen chronischer Krankheiten oder mysteriöse neue Krankheitsmerkmale vor (Ausschläge, Fieber, neuralgische Schmerzen).

Die Kerkermeister sind sich dessen bewußt, daß einzig und allein während der Besuche und bei der Korrespondenz der Gefangene die Integrität seiner Persönlichkeit wiederherstellt und neue Kraft aufnimmt.

Deshalb wird auch die Korrespondenz beschränkt. Seit dem Jahre 1973 ist ein Brief wöchentlich, geschrieben an eine Adresse, bewilligt. Die Briefe werden den Gefangenen oft zurückgegeben, damit sie sie neu schreiben (wegen eines "anstößigen « Satzes, wegen der »unansehnlichen « Schrift). Die Briefe, die von uns, den Familienangehörigen, übersandt werden, werden manchmal beschlagnahmt, ohne daß sie unser gefangengehaltener Verwandter durchlesen könnte. Manche Briefe gehen auf unerklärliche Weise verloren. Die Zensur ist unwürdig und unsinnig. Man darf nicht über ein Buch, einen Film, über die Arbeit des Gefangenen, noch über die Arbeit seiner Verwandten auf freiem Fuß schreiben, man darf keine Verse, Betrachtungen aufschreiben, es dürfen im Brief keine Namen, nicht einmal die Namen von Autoren, sofern es sich um Tschechen handeit, vorkommen. Wenn der Brief durch die Zensur trotzdem zum Gefangenen gelangt, wird er ihm beim Eintreffen des nachfolgenden Briefes abgenommen und wird vernichtet, obwohl der Gefangene das Recht besitzt, den Brief zu behalten (mindestens bei seinen persönlichen Sachen, die außerhalb der Zelle aufbewahrt werden). Gegen das Vorgehen der Zensur gibt es keine Berufung.

Die Familie des Häftlings wird materiell spürbar geschädigt. In der Mehrzahl der Fälle verliert sie den hauptsächlichen Ernährer. Die Ehegattinnen, die Mütter und andere Verwandten wurden durch Kündigungen ihrer Arbeitsplätze oder durch die Verschlechterung des sozialen Status bei ihrer Beschäftigung betroffen. Die Kinder werden gehindert, auf Mittel- und Hochschulen zu studieren. Sie werden so in mehreren grundlegenden Bedürfnissen beschränkt, wodurch ihr Lebensweg unausweichlich markiert wird. Obwohl sie völlig schuldlos sind, werden sie für ihr ganzes zukünftiges Leben bestraft. Durch die Diskriminierung der Kinder wird die Verfassung der CSSR und der Internationale Pakt über bürgerliche und politische Rechte verletzt.

Besonderer Status tschechischer politzscher Häftlinge und das System der Druckausübung

In einem anderen Land oder in einer anderen Zeit würde man unsere eingekerkerten Verwandten offiziell "politische Häftlinge« nennen, weil sie wegen der Tätigkeit oder wegen der Haltung im politischen Bereich eingekerkert wurden, nämlich wegen der Verteidigung der Bürgerrechte. Der Begriff "politischer Häftling « er- 
möglicht dann den Vergleich mit der Lage in anderen Zeiten und in anderen Ländern. Die Rechtsgefühle der gegenwärtigen Offentlichkeit unterscheiden nämlich die politischen Häftlinge von den kriminellen Verbrechern und setzen für die "politischen « würdige Haftbedingungen voraus, die ihrer Kultur und der ethischen Motivation entsprechen.

In der CSSR wird der Begriff "politischer Häftling « mit dem Verweis darauf nicht anerkannt, daß bei uns nur "Inhaftierte nach Kapitel I des besonderen Teiles des Strafgesetzes " existieren. Unsere Verwandten genießen also keine Vorrechte, die in den übrigen gesitteten Ländern inhaftierten politischen Gegnern gewährt werden. Unsere Verwandten genießen jedoch nicht einmal die schwachen Rechte der kriminellen Häftlinge - ja sie werden viel schlimmer behandelt als diese.

Für die gegenwärtigen tschechischen politischen Häftlinge wurde ein völlig außerordentlicher Status geschaffen. Die besondere Anordnung dafür kennen wir nicht, wenn wir jedoch verfolgen, wie sie in der Praxis durchgeführt wird, beobachten wir, daß sie den höheren Rechtsnormen und schließlich auch der Verfassung der CSSR widerspricht.

Für diesen besonderen Status trägt das System der Einkerkerung tschechoslowakischer politischer Häftlinge noch einige besondere Züge:

Die Unterlassung grundlegender Fürsorge ist die weniger augenfällige Art, die aber nicht minder grausam als die direkte physische Aggression ist. In der Lage der völligen Machtlosigkeit und des Darbens gewinnen normale Gegenstände und Erscheinungen plötzlich die Bedeutung des Lebens oder des Todes. Eine zu schwache Glühbirne in einer finsteren Gefängniszelle, der Arbeitsschemel ohne eine Rückenlehne, multipliziert mit den zehn Stunden eines jeden Tages und dann mit den Tagen, Wochen, Monaten, Jahren verwandeln sich in Torturinstrumente. Ein Kranker mit fieberhaften Schwellungen und Geschwüren auf dem ganzen Körper (infolge einer nicht behandelten Avitaminose) muß sich auf einem absichtlich harten Lager, das ihm zugewiesen wurde, wie auf einem Quälinstrument vorkommen.

Intellektuelle Isolierung bedeutet psychische Tortur. Bei einer starken Beschränkung der Eindrücke der Sinne in der tagtäglichen Monotonie der Gefängniszelle fühlt der Mensch besonders stark das Bedürfnis nach Anregungen, nach Informationen, nach Bildern, die aus der äußeren Welt kommen. Der Geist unserer eingekerkerten Verwandten wird jedoch absichtlich abgestumpft und sogar abgetötet.

In manchen Gefängnissen dürfen die Hälftlinge nur eine Zeitung und ein Buch wöchentlich aus der Gefängnisbibliothek nach Wahl des Gefängnisaufsehers erhalten. Es kommt vor, daß dasselbe Buch einigemale der gleichen Gefängniszelle zugeteilt wird. (Es überwiegen stalinistische Autoren und tschechische Klassik des 19. und des Anfangs des 20. Jahrhunderts.) Unseren Verwandten wird es unmöglich gemacht, jedwede Fach- und auch Fremdsprachenliteratur zu lesen oder aus Fremdsprachenlehrbüchern und Wörterbüchern zu lernen. Es wurde kategorisch abgelehnt, ihnen sogar die marxistische Literatur auszuleihen. Es ist ihnen verboten, sich irgendwelche Aufzeichnungen, Konzepte, Exzerpte, Ubersichten von fremdsprachigen Wörtern anzufertigen. Alles wird Ihnen beschlagnahmt. Es wird völlig verhindert, sich wenigstens in der sogenannten Freizeit das Fachwissen ihres eigenen Berufes zu erneuern oder aufrechtzuerhalten.

Ein Bestandteil der Gefängniskultur ist das zwangsweise Abhören des Drahtrundfunks. Einmal innerhalb von I4 Tagen wird der Häftling für zwei Stunden in die Gefängniszelle für das »kulturelle Ausleben« geführt, wo es ein Tischlein, vier Stühle und einen Fernsehapparat gibt. Die festgesetzte Zeit des "Auslebens w wird strikt eingehalten - sobald dies um ist, wird der Häftling hinausgeführt. Er sieht also im Fernsehen einige unbeendete Teile verschiedener Programme. 
Unsere Verwandten sind zu einer schöpferischen Untätigkeit verurteilt und angesichts der Abhörapparatur in den Zellen sind sie in langen unendlichen Stunden nur ihren eigenen Gedanken überlassen. Dadurch wird die Verfassung der CSSR und das Gesetz über den Strafvollzug verletzt.

Absichtliches Schikaneren und Willkürakte gehören zu der Eigeninitiative einzelner Angehöriger des Gefängnispersonals. Im Vergleich mit den frei seitens der Kerkermeister durchgeführten Ungerechtigkeiten konstrasiert die Grausamkeit der Strafen, denen die Häftlinge unterworfen werden. Außer der komplexen Strafe des Freiheitsentzuges werden die Hälftlinge Verschärfungen unterworfen, und zwar wegen der kleinsten »Vergehen«. Politische Häftlinge werden grob angeschrieen und in ihren Rechten beschränkt. Das gesamte System des zwangsweisen Lebens soll den Gefangenen ununterbrochen in einer Spannung und im Bewußtsein der Rechtlosigkeit und der Auslieferung an die unbeschränkte Willkür halten.

Das Bewußtsein der Absurdität vernichtet das geistige Leben des Häftlings vielleicht am meisten. Es steht im Widerspruch mit der menschlichen Existenz als solcher. Der Mensch realisiert seine Menschlichkeit damit, daß er die Frage nach dem Sinn seines Geschlechtes und seiner selbst stellt. Diese Frage wird durch denkende Menschen aller Zeiten gestellt und sie bildet auch die allgemeinste Grundlage des Entscheidens und der Taten unserer Verwandten, die zu politischen Gefangenen gemacht wurden. Das Bewußtsein des Zieles und die Erkenntnis des Sinnes motiviert ihr Sein. Demgegenüber ist die absurde Existenz ein Faktor, der sich gegen das Wesen des Lebens stellt. Wenn ein Sinn der täglichen Tätigkeit fehlt, verfällt der Mensch in die Depression, sein Geist siecht dahin. Die Verletzung der Menschenrechte ist offensichtlich.

\section{Beschwerden, Verteidigung}

Geleitet durch grundsätzliches Vertrauen zum Menschen setzen wir voraus, daß es auch unter dem Personal des Gefängnisses oder unter den für das Gefängniswesen verantwortlichen Personen Menschen gibt, bei denen die Objektivität die ungerechte Voreingenommenheit überwiegt. Wir befürchten jedoch, daß der umgekehrte Fall öfter vorkommt, und vor allem, daß es gegen ihn keine Verteidigung gibt, daß er zum Bestandteil des Systems wurde.

Es wurden bereits mehrere Beschwerden über das Schicksal tschechischer politischer Häftlinge eingereicht. In den meisten Fällen wanderten die Beschwerden von den höchsten Stellen, an die sie bestimmt waren, zur Erledigung zurück zu denjenigen Stellen, über die die Beschwerden verfaßt wurden.

Nach wie vor dauert die grundlegendste Ungerechtigkeit an, daß nämlich diejenigen, die eingekerkert sind, weder Verbrecher noch Terroristen sind.

Eingekerkert sind rechtschaffene und gebildete Leute dafür, daß sie Bürgerrechte für sich und für ihre Mitbürger forderten, und zwar mit Mitteln, die der internationalen Auffassung der Menschenrechte entsprechen, die unsere Regierung zu respektieren in Helsinki versprach.

Obwohl unsere Verwandten durch diese grausame Ungerechtigkeit getroffen wurden, obwohl sie unter vernichtenden Bedingungen leben, obwohl sie täglich mit der Macht Einzelner konfrontiert werden, die sich ihnen weder mit Intellekt noch mit der Moral gleichstellen können - erniedrigten sie sich moralisch nicht, brachten es fertig, Ruhe, Solidarität, menschliche Würde, Tapferkeit und Treue den Idealen der Demokratie, des Humanismus, des Sozialismus zu bewahren.

Im Einklang mit dem internationalen Recht, im Einklang mit der Schlußakte der Konferenz über die Sicherheit und Zusammenarbeit in Europa, die in Helsinki auch für die Tschechoslowakische Sozialistische Republik unterschrieben wurde, fordern 
wir die sofortige Freilassung unserer Verwandten, tschechischer politischer Häft-

linge.

Prag - Brünn, den r. März 1976.

Unterschrieben:

Vilém Müller

Frantiska Müllerová

Marie Rusková

Ivan Rusek

Tatána Rusková

Rudolf Sabata

Ruzena Sabatová

Anna Sabatová

Jan Sabata

Václav Sabata

Eliska Skrenková (Hüblová)

Jana Tesarová

Brno, Jana Babáka 3/5

Brno, Jana Babáka 3/5

Brno, Drevarská 20

Brno, Drevarská 20

Brno, Drevarská 20

Dolenice c. 2

Dolenice c. 2

Praha 2, Anglická 8

Brno, nám. 28. rijna 20

Brno, Slovanská 5

Praha 5, Na Brezince I4

Praha 4, Pankrác II, is 50

\section{Demonstrationsrecht und polizeiliche Überwachung}

Der Staatsapparat hat seit jeher versucht, seine Kontroll- und Steuerungsmechanismen schon bei der politischen Meinungsbildung und Meinungsäußerung des Bürgers einzusetzen. Neuestes Beispiel dafür sind die in letzter Zeit zunehmenden polizeilichen Überwachungen politischer Demonstrationen selbst dann, wenn sie ordnungsgemäß angemeldet worden sind und "störungsfrei « verlaufen.

Dies geschah auch in dem folgenden von den Gerichten zu entscheidenden Fall: Mitte 1973 fand in Tübingen eine Demonstration von etwa 300 Personen gegen die geplante Änderung eines Hochschulgesetzes statt. Die Versammlung, die ordnungsgemäß angemeldet war und »störungsfrei« verlief, wurde von Kriminalbeamten überwacht und fotografiert. Eine Gruppe von Demonstranten, unter ihnen der Angeklagte, entdeckte einen fotografierenden Polizeibeamten und eilte zu ihm. Als der Polizeibeamte nach dem Eintreffen der Gruppe die Herausgabe des Films verweigerte, ergriff der Angeklagte den Fotokoffer und entfernte sich.

Das LG Tübingen' erblickte erst in dem späteren Behalten des Koffers ein strafbares Verhalten; die vorangegangene Wegnahme des Koffers sei dagegen durch Notwehr gerechtfertigt gewesen, weil der Polizeibeamte die Teilnehmer der Versammlung ohne gesetzliche Grundlage und unter Verletzung von Grundrechten (Art. 8 I GG) fotographiert hätte.

Dagegen ist nach dem $\mathrm{BGH}^{2}$ die Annahme von Notwehr rechtsfehlerhaft, weil das LG nicht berücksichtigt habe, daß die Polizeibeamten als Grund für das Fotografieren angaben, "man habe mit Hilfe der Lichtbilder die - unbekannten - Täter früherer Wandschmierereien und Vorlesungsstörungen, die als Teilnehmer an der Demonstration vermutet wurden, identifizieren wollen.« Unter diesen Umständen

\footnotetext{
Das Urteil des LG Tübıngen vom 18. 3. 1974 ist abgedruckt in: KJ 74, 4 I 8 ff. mit einer Anmerkung von Werner Robbers, $\mathrm{KJ} 74,425 \mathrm{ff}$.

$2 \mathrm{BGH}, \mathrm{NJW} 75,2075 \mathrm{f}$. und JZ 76, $31 \mathrm{f}$. mit einer Anmerkung von Walter Schmidt, JZ $76,32 \mathrm{f}$. Die Anmerkung yon W Robbers in der KJ wurde weder vom BGH noch von W Schmidt zur Kenntnis genommen.
} 